\title{
O USO DE MODELOS MATEMÁTICOS NA ENGENHARIA PARA DISCUSSÃO DE QUESTÕES SOCIOCIENTÍFICAS: VARIÁVEIS SOCIAIS E HUMANAS
}

\author{
The Use of Mathematical Models in Engineering to Discuss Socio-Scientific Issues: \\ Social And Human Variables
}

\author{
Stefane Layana Gaffuri* \\ Leandro Bordin** \\ Paula Andrea Grawieski Civiero*** \\ Walter Bazzo ${ }^{* * * *}$
}

\begin{abstract}
Resumo: O artigo tem como objeto de estudo o modelo gaussiano de dispersão de poluentes atmosféricos, usualmente praticado no ensino de engenharia. Para além das técnicas, busca-se discutir as questões sociocientíficas ao considerar as variáveis sociais e humanas que constituem tal modelo, com o objetivo de conduzir o estudante a uma visão crítica na identificação e resolução de problemas. Este estudo faz uso de conceitos matemáticos, físicos e químicos, sendo um trabalho interdisciplinar entre as ciências básicas. O modelo em questão equaciona variáveis técnicas e, com isso, proporciona aos futuros profissionais a possibilidade de simular o comportamento da nuvem de dispersão de acordo com a alteração de qualquer uma dessas variáveis. Decisões podem ser tomadas a partir de mudanças de cenário e, portanto, a engenharia cumpre com seu papel de resolver problemas, como na indústria que, no seu fazer produtivo e tecnológico, causa um problema ambiental para a sociedade. Por isso, conclui-se ser necessário desvelar as variáveis contemporâneas que compõem esse modelo ao debater sobre as implicações sociais da tecnociência que estão imbricadas na resolução deste. Trabalhar nessa perspectiva é explorar ao máximo o potencial da matemática na educação em engenharia.
\end{abstract}

Palavras-chave: Matemática. Engenharia. Questões sociocientíficas. Variáveis contemporâneas.

\footnotetext{
* Licenciada em Matemática, Mestra em Ensino de Física e Matemática, Doutorado em andamento em Educação Científica e Tecnológica (PPGECT/UFSC). Professora da Universidade Tecnológica Federal do Paraná (UTFPR) - Câmpus Francisco Beltrão/PR. Orcid: https://orcid.org/0000-0002-9400-5210. Email: stefanegaffuri@utfpr.edu.br

** Engenheiro Civil, Mestre em Engenharia, Doutor em Educação Científica e Tecnológica (PPGECT/UFSC). Professor Adjunto da Universidade Federal da Fronteira Sul (UFFS) - Câmpus Chapecó/SC. Orcid: https://orcid.org/0000-0002-4703-6671. E-mail: lbordin@uffs.edu.br

*** Licenciada em Matemática (FAFI), Mestra em Ensino de Matemática (UFRGS), Doutora em Educação Científica e Tecnológica (UFSC). Professora do Instituto Federal Catarinense (IFC) - Câmpus Rio do Sul. Membro do Núcleo de Estudos e Pesquisas em Educação Tecnológica (NEPET/UFSC). Orcid: https://orcid.org/0000-00025841-7330. E-mail: paula.civiero@ifc.edu.br

***** Engenheiro Mecânico, Mestre em Engenharia Mecânica, Doutor em Educação. Professor Titular da Universidade Federal de Santa Catarina (UFSC), Coordenador do Núcleo de Estudos e Pesquisas em Educação Tecnológica (NEPET), Professor no Programa de Pós-Graduação em Educação Científica e Tecnológica (PPGECT/UFSC). Orcid: https://orcid.org/0000-0003-0093-8229. E-mail: walter.bazzo@ufsc.br
} 
Abstract: The article has as object of study the Gaussian model of dispersion of atmospheric pollutants, usually practiced in the teaching of Engineering. In addition to the techniques, we seek to discuss socio-scientific issues, when considering the social and human variables that constitute such a model, with the aim of leading the student to a critical view in the identification and resolution of problems. This study makes use of mathematical, physical, and chemical concepts, being an interdisciplinary work among the basic sciences. The model in question equates technical variables and thereby provides future professionals with the possibility to simulate the behavior of the dispersion cloud according to the alteration of any one of these variables. Decisions can be made based on changes in the scenario and, therefore, Engineering fulfills its role of solving problems, in this case of the industry that in its productive / technological way causes an environmental problem for society. Therefore, it is concluded that it is necessary to unveil the contemporary variables that make up this model when discussing the social implications of technoscience that are interwoven in its resolution. Working from this perspective is to exploit the potential of mathematics in engineering education to the full.

Keywords: Mathematics. Engineering. Socioscientific Issues. Contemporary variables.

\section{Introdução}

O uso intensivo de ciência e tecnologia e a formação de profissionais altamente qualificados são essenciais à visão da educação em engenharia, no que diz respeito às questões tecnológicas da profissão. Frente a isso, o conceito de qualificação profissional está atrelado às capacidades de coordenar informações, interagir em grupos de pessoas, interpretar a realidade e não se limitar a problemas teóricos.

Quando se analisa o perfil do egresso preconizado pelas Diretrizes Curriculares Nacionais para os cursos de engenharia (DCNs), percebe-se a preocupação com uma formação de engenheiro um tanto mais 'alargada' que a puramente técnica. Segundo o parecer homologado recentemente, a educação em engenharia vem se atualizando ao longo dos anos e, por isso, estabelece que o perfil do egresso "deve se voltar para uma visão sistêmica e holística de formação, não só do profissional, mas também do cidadão-engenheiro, de tal modo que se comprometa com os valores fundamentais da sociedade na qual se insere" (PARECER CNE/CES n. 1/2019, p. 25).

Conforme o parecer, estimula-se a formação de um profissional tanto técnica como humana, visto que se exige uma formação que se comprometa com valores fundamentais. Sobre isso, Santos e Mortimer (2009, p. 192-193) afirmam que

[...] uma educação científica e tecnológica humanística buscaria incorporar ao currículo discussões de valores e reflexões críticas que possibilitassem desvelar a condição humana. Não se trata de fazer uma educação contra ou a favor do uso da tecnologia, mas de uma educação em que os alunos possam refletir sobre a sua condição no mundo frente aos desafios postos pela ciência e tecnologia.

Ao refletir sobre esse contexto, questiona-se: como os educadores em engenharia podem contribuir para a formação desse engenheiro? Para tanto, o parecer define também o desenvolvimento de competências associadas aos seguintes princípios: 
I. Formular e conceber soluções desejáveis de Engenharia, analisando e compreendendo a necessidade dos usuários e seu contexto;

II. Analisar e compreender os fenômenos físicos e químicos por meio de modelos simbólicos, físicos e outros, uma vez verificados e validados por experimentação;

III. Conceber, projetar e analisar sistemas, produtos (bens e serviços), componentes ou processos;

IV. Implantar, supervisionar e controlar as soluções de Engenharia;

V. Comunicar-se eficazmente nas formas escrita, oral e gráfica;

VI. Trabalhar e liderar equipes multidisciplinares;

VII. Conhecer e aplicar com ética a legislação e os atos normativos no âmbito do exercício da profissão;

VIII. Aprender de forma autônoma e lidar com situações e contextos complexos, atualizando-se em relação aos avanços da ciência, da tecnologia, bem como em relação aos desafios da inovação. (PARECER CNE/CES n 1/2019, p. 25).

Ao se considerar esses itens, constata-se a importância de discutir, além das técnicas, também as questões sociocientíficas, principalmente as variáveis sociais e humanas que constituem tal modelo, com o objetivo de conduzir o estudante de engenharia a uma visão crítica na identificação e resolução de problemas. Segundo Reis (2006, p. 66), essas questões são

[...] relativas às interações entre ciência, tecnologia e sociedade (nomeadamente, as polêmicas despoletadas pelos eventuais impactos sociais de inovações científicas e tecnológicas), que dividem tanto a comunidade científica como a sociedade em geral, e para as quais diferentes grupos de cidadãos propõem explicações e tentativas de resolução incompatíveis, baseadas em valores alternativos. Essas questões sociocientíficas possuem uma natureza contenciosa, podem ser analisadas segundo diferentes perspectivas, não conduzem a conclusões simples e envolvem, frequentemente, uma dimensão moral e ética.

Para Vallim, Farines e Cury (2006), as transformações tecnológicas, as divisões de trabalho e as variações bruscas na economia colocam engenheiros frente a situações nas quais devem solucionar problemas cada vez mais estruturados e complexos, em um ambiente em constante transformação. No entanto, nota-se que, ao se deparar com um problema real a ser solucionado, dificilmente o indivíduo consegue realizar uma conexão entre o que estudou e o problema. Para os autores, essa dificuldade está na percepção da oportunidade de aplicar os conceitos teóricos ou em "[...] fazer as adaptações necessárias para que um problema real possa ser tratado por algum conhecimento teórico pertinente" (VALLIM; FARINES; CURY, 2006, p. 1).

A matemática é uma ciência construída socialmente e está presente em diversos ramos da atividade humana, contribuindo para a formação subjacente dos constructos científicos e tecnológicos. Assim, há uma urgência em repensar esse trio: matemática, tecnologia e engenharia; principalmente quanto às imbricações da tecnociência no ensino de matemática e na formação dos engenheiros, "ao considerar que é preciso capacitar os cidadãos com competência crítica para questionar as decisões tecnocientíficas, bem como participar ativamente delas" (CIVIERO, 2016, p. 243).

Tais argumentos ganham força posto que as variáveis que definem os problemas de engenharia não são - ou, pelo menos, não deveriam ser - meramente técnicas. Para além das dificuldades com a aplicação dos conceitos e ferramentas matemáticas na resolução de problemas de engenharia, precisa-se questionar a pouca ênfase dada às questões sociocientíficas, em especial, àquelas que abrangem variáveis de cunho social que podem - e 
devem - ser incorporadas nas soluções tecnológicas. Tais variáveis contemporâneas "surgem a todo instante em uma civilização que está vulnerável às mais aceleradas mutações em seu comportamento cotidiano" (BAZZO, 2019, p. 21).

Atrelado a esse contexto, pensou-se em trabalhar uma situação hipotética e um modelo matemático já conhecido: o modelo gaussiano da dispersão de poluentes na atmosfera. A escolha se deve ao fato de o controle da poluição atmosférica, em escala global, ser usualmente realizado por meio de uma rede de monitoramento da qualidade do ar. Essa rede permite zelar pela segurança e saúde humana e ambiental, bem como, predispor de intervenções específicas caso haja uma superação dos níveis limites estabelecidos pela legislação (MOREIRA; TIRABASSI, 2004).

No entanto, por motivos de caráter administrativo e econômico, muitas vezes os pontos de medida dessa rede são limitados, podendo ser pouco representativos. Sendo assim, para Moreira e Tirabassi (2004, p. 169),

[...] os modelos matemáticos que simulam o transporte e a difusão dos poluentes na atmosfera constituem uma importante ferramenta para auxiliar as medidas de concentrações e saber a evolução das mesmas. [...] Somente com modelos matemáticos é possível fazer previsões ou simular campos de concentração em conexão com políticas de limitação da liberação de poluentes em concordância com planos de melhoria da qualidade de vida da população.

Com a compreensão de que os modelos matemáticos são representações de parte da realidade, pretende-se - por meio do modelo matemático da dispersão de poluentes atmosféricos, usualmente praticado no ensino de engenharia - problematizar a urgência de discutir o modelo, as questões sociocientíficas e as variáveis contemporâneas que constituem tal modelo. Dessa maneira, o estudante em engenharia seria conduzido a uma visão crítica e criativa na identificação e resolução de problemas.

\section{0 ensino de matemática na engenharia}

As dificuldades enfrentadas pelos ingressantes nos cursos de engenharia estão geralmente vinculadas às disciplinas das áreas básicas, entre elas a Matemática. Não se pode negar que há uma preocupação sobre o ensino de matemática nos cursos de engenharia. Um dos motivos está no elevado índice de retenção e evasão dos estudantes nesses cursos. Pesquisas mostram que a reprovação nas disciplinas básicas pode estar vinculada a não conclusão do curso. (GODOY; ALMEIDA, 2017; GOMES, 2009; GOMES, 2015; GUNTHER, 2016).

Godoy e Almeida (2017) investigaram a importância das disciplinas da área de Matemática no fracasso escolar dos estudantes do curso de engenharia. Para isso, selecionaram 83 trabalhos apresentados no Congresso Brasileiro de Educação em Engenharia (COBENGE), no período compreendido entre os anos de 2000 a 2014. Os autores perceberam que as disciplinas da área de Matemática e das Ciências Naturais estão vinculadas à fraca formação dessas mesmas áreas na Educação Básica, de modo a contribuir, consideravelmente, para a evasão. A partir dessa constatação, os trabalhos pesquisados apresentam propostas para combater, direta ou indiretamente, os altos índices.

Percebe-se que há um avanço nas discussões relacionadas ao ensino de matemática nos cursos de engenharia e um consenso de que, além do conhecimento matemático, é necessário 
incorporar teorias associadas à educação; apresentar contextos significativos aos alunos; usar, sobremaneira, as ferramentas computacionais para construir o conhecimento matemático; e, por fim, acolher os alunos, tratando de oportunizar ambientes onde eles possam preencher as lacunas conceituais advindas de uma formação educacional básica deficiente. Porém, nota-se que as pesquisas focam em 'inovar' o ensino de matemática mediante as metodologias ou o uso de recursos tecnológicos com o intuito de melhorar a qualidade do ensino-aprendizagem e evitar a evasão dos cursos. Será esse o caminho?

Insiste-se em tecer novos caminhos para a educação tecnológica, em especial para o ensino de matemática na engenharia, de modo a criar situações e subsidiar contextos apropriados para os alunos terem uma formação mais concreta. Sendo assim, a discussão de questões sociocientíficas propicia aos estudantes perceber que a ciência é uma construção humana cultural e temporal, influenciada pela sociedade. Isso está de acordo com o que consideram Bazzo, Pereira e Linsingen (2016, p. 191):

[...] questões contemporâneas que afligem estudantes e profissionais da área quase nunca são refletidas ou discutidas como parte integrante da formação de cidadãos engajados num determinado contexto social e histórico. Isso provoca uma lacuna que entrava ou, antes, suprime as capacidades reflexiva e analítica tão requisitadas numa sociedade globalizada.

O uso de modelos matemáticos pode auxiliar na discussão dessas questões, visto que eles ajudam na problematização e no questionamento da realidade. Além disso, esse debate tem grande potencial para desenvolver competências e habilidades relevantes para a cidadania, conforme preconizam as DCNs. Entre elas, pode-se citar: a competência para pesquisar informações e reconhecer as incoerências entre os dados; o desenvolvimento do trabalho cooperativo e a capacidade de argumentar e escutar o que o outro tem a dizer; o estímulo da independência intelectual; a reavaliação de diferentes opiniões; o entendimento dos diversos aspectos envolvidos em um assunto, entre outros (REIS, 2006, 2007).

\section{Modelagem matemática em engenharia}

Ao pensar no ensino de matemática em engenharia, é basilar ter a compreensão de que o desenvolvimento tecnocientífico tem assegurado distintas transformações na sociedade. Nesse sentido, ao eleger um modelo matemático a ser desenvolvido, é imprescindível provocar a construção crítica de conhecimentos que possam romper com limites do conhecimento matemático específico e estritamente técnico, favorecendo as reflexões sobre as questões sociocientíficas ao considerar-se as variáveis contemporâneas imbricadas ao modelo matemático. Skovsmose (2001) corrobora essa compreensão ao discutir a importância da crítica ao modelo matemático e ao considerar que a matemática intervém na realidade e a formata. Por sua vez, muitos modelos matemáticos são utilizados para alterar comportamentos.

A matemática intervém na realidade ao criar uma "segunda natureza" ao nosso redor, oferecendo não apenas descrições de fenômenos, mas também modelos para a mudança de comportamentos. Não apenas, "vemos" de acordo com a matemática, nós "agimos" de acordo com ela. As estruturas matemáticas vêm a ter um papel na vida social tão fundamental quanto o das estruturas ideológicas na organização da realidade (SKOVSMOSE, 2001, p. 83).

Nesse contexto, ao compreender a relação de imanência entre a tecnociência e a matemática, faz-se necessário debater sobre as implicações sociais dos modelos matemáticos 
na sociedade tecnológica (CIVIERO, 2016). Entretanto, para que a matemática na educação em engenharia possa contribuir como um instrumento para a interpretação da realidade, é preciso ultrapassar a linha tênue, porém, tradicional, do 'como fazer' e passar a indagar 'para quê?', 'para quem?' e 'por quê?' desse modelo.

Segundo Civiero (2016, p. 29), a matemática

\begin{abstract}
pode contribuir para a formação de sujeitos críticos que participam das discussões e que são capazes de compreender a real situação e tomar decisões voltadas para a melhoria da qualidade de vida. Pessoas que se indaguem quanto ao porquê, para que e para quem estão a tomar suas decisões, a destinar suas invenções e a protagonizar sua existência.
\end{abstract}

Dessa maneira, a discussão de questões sociocientíficas ligadas ao conhecimento matemático pode ser um artifício potencializador da educação comprometida com os processos sociais; uma educação que instiga o questionamento crítico dos modos de pensar, agir e viver tecnicistas que, cada vez mais, se impõem como únicos e verdadeiros. A escolha do modelo em questão equaciona variáveis como direção e velocidade do vento, classe de estabilidade da atmosfera, altura da fonte de poluição, taxas de emissão e superfície do solo. Dessa forma, proporciona aos profissionais de engenharia a possibilidade de simular o comportamento da nuvem de dispersão de acordo com a alteração de qualquer uma de tais variáveis. As melhores decisões técnicas podem ser tomadas a partir dessas mudanças de cenário. Portanto, a engenharia cumpre com seu papel de resolver problemas, em especial, no que se refere às indústrias, pois no seu fazer produtivo/tecnológico causam problemas ambientais para o coletivo da sociedade.

\title{
40 modelo da dispersão de poluentes na atmosfera
}

Os modelos de dispersão atmosférica são ferramentas para monitorar os poluentes atmosféricos. Estes podem ter diferentes finalidades, entretanto, para a verificação da dispersão de poluentes emitidos por fontes lineares, diversos modelos matemáticos vêm sendo utilizados, entre eles, o modelo de dispersão gaussiana - nosso objeto de estudo.

De maneira geral, os dados de entrada para a maioria desses modelos são a direção e a velocidade do vento, a classe de estabilidade da atmosfera, a altura da camada da mistura, as taxas de emissão e a superfície do solo.

Os objetivos do uso de modelos de dispersão atmosférica são:

a) avaliação da eficiência de técnicas e estratégias propostas para o controle das emissões;

b) estudo dos impactos ambientais (EIA) para um novo empreendimento;

c) determinação de responsabilidades frente aos níveis atuais de poluição;

d) planejamento da ocupação territorial urbana.

O estudo dos impactos ambientais e avaliação da qualidade do ar, geralmente, está relacionado com duas etapas: (1) empregar modelos de avaliação simplificados para se ter uma rápida estimativa se a fonte de emissão certamente causará um problema de poluição do ar, ou se a fonte tem o potencial de causar um problema de qualidade do ar. (2) Se os resultados da avaliação simplificada revelarem que existe um potencial problema, métodos mais avançados 
de avaliação devem ser utilizados (como modelos mais avançados e maior atenção aos dados de entrada).

Para problematizar, supõe-se que um meio atmosférico esteja sendo poluído por uma chaminé. Essa fonte está lançando poluentes continuamente, e eles irão se dispersar no ar, resultando na formação de uma pluma (nuvem). Como analisar o comportamento dessa pluma? Estudar o comportamento da pluma significa estudar como o meio atmosférico transporta e dispersa os poluentes nele lançados. O comportamento da pluma depende principalmente do clima da região, do movimento dos ventos (que depende de forças de pressão e de atrito e da topografia), da superfície do solo e da sua ocupação (por edifícios, por exemplo) (BRAGA et al., 2005).

O modelo de dispersão gaussiano é utilizado para explicar o comportamento da pluma em períodos de tempo relativamente pequenos. A variação da concentração de poluentes ao nível do solo, à medida que se afasta da fonte emissora, pode ser expressa por uma curva gaussiana. No cálculo do modelo em questão, a pluma sofre dispersão em planos horizontais e verticais tomando a forma de uma curva de Gauss, com um máximo no centro da pluma. A distribuição da concentração da pluma ao redor do eixo central pode ser considerada uma gaussiana com valores de distribuição sendo considerados afastamentos do eixo da pluma. $\mathrm{Na}$ Figura 1, apresenta-se uma representação da dispersão de uma pluma segundo uma distribuição gaussiana.

Figura 1 - Ilustração do sistema de coordenadas utilizado na descrição da pluma gaussiana

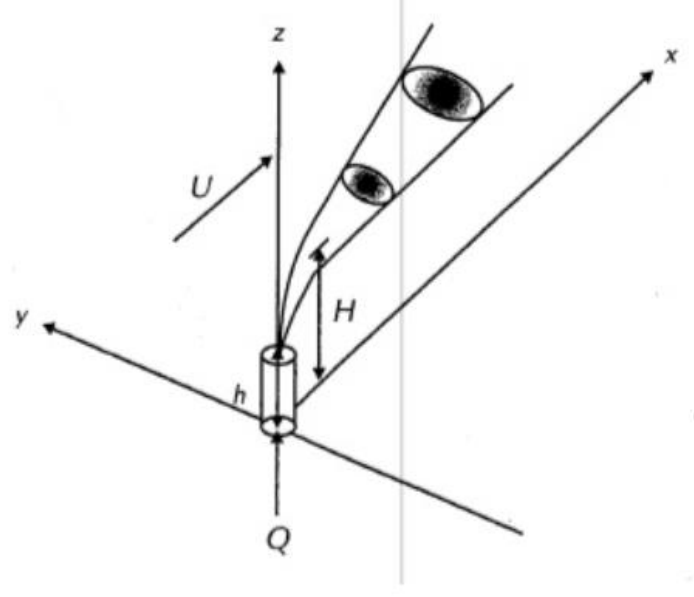

Fonte: Braga et al. (2005).

A Figura 1 esquematiza como uma fonte e sua emissão são consideradas. A pluma, ao sair da chaminé, eleva-se por causa de sua temperatura e da temperatura ambiente (cuja diferença provoca empuxo) e velocidade de saída. Ao ser lançada na atmosfera, a pluma sofre efeito de dispersão de acordo com parâmetros meteorológicos, tais como velocidade e direção do vento, temperatura, estabilidade atmosférica etc. O resultado é um processo de advecção (transmissão do calor pelo deslocamento de massa atmosférica no sentido horizontal) pelo vento médio (que transporta para onde o vento está soprando) e de difusão turbulenta ('abertura' horizontal e vertical da pluma). Esse processo pode ser representado por equações matemáticas e as concentrações resultantes no entorno da fonte (com coordenadas $\mathrm{x}$, y e $\mathrm{z}$ ) que podem ser 
calculadas, em função dos aspectos meteorológicos e topográficos, obviamente devido às características das emissões.

Braga et al. (2005) explicam que, como resultado, há uma equação (1) que relaciona as concentrações de um poluente com as coordenadas espaciais, com o tempo e com outros poluentes que possam interferir no poluente em análise, como nos casos de poluentes reativos na atmosfera.

$$
C_{(x, y, z)}=\frac{Q}{2 \pi u \sigma_{y} \sigma_{z}} \cdot \exp \left(-\frac{y^{2}}{2{\sigma^{2}}_{y}}\right) \cdot\left[\exp \left(-\frac{(z-H)^{2}}{2{\sigma^{2}}_{z}}\right)+\exp \left(-\frac{(z+H)^{2}}{2{\sigma^{2}}_{z}}\right)\right]
$$

Onde,

C é a concentração média do poluente analisado na posição $\mathrm{x}, \mathrm{y}$ e $\mathrm{z}\left(\mathrm{g} / \mathrm{m}^{3}\right)$;

$\mathrm{Q}$ é a concentração de emissão em um determinado período $(\mathrm{m} / \mathrm{t})$;

H é a altura efetiva da chaminé (m);

u é a velocidade média do vento $(\mathrm{m} / \mathrm{s})$;

$\sigma_{y}$ é o coeficiente de dispersão horizontal em velocidade constante de vento;

$\sigma_{z}$ é o coeficiente de dispersão vertical $(\mathrm{m})$;

$\mathrm{x}, \mathrm{y}, \mathrm{z}$ são as coordenadas cartesianas do ponto.

Ao trabalhar com o modelo, deve-se considerar algumas hipóteses (TURNER, 1994, apud LISBOA, 2010):

a) não se considera a deposição de material e reações de superfície;

b) a emissão dos poluentes é considerada uniforme no tempo;

c) a direção e a velocidade do vento são constantes no período de tempo considerado;

d) não são consideradas as reações químicas na atmosfera;

e) a pluma gaussiana assume que o vento tem intensidade e direção constantes em um intervalo de tempo;

f) a área considerada deve apresentar um terreno homogêneo (relativamente plano);

g) a equação gaussiana traduz uma situação estacionária, isto é, a emissão de poluentes e os parâmetros meteorológicos são constantes;

h) o valor de $\mathrm{H}$ não é igual ao valor da altura (h) da chaminé.

Para as hipóteses apresentadas, segundo o autor, os cálculos de concentração são razoáveis para períodos variando de 10 minutos a 1 hora.

Segundo Lisboa (2010), a caracterização da altura de subida da pluma em termos das propriedades dos gases emitidos e do estado atmosférico é um problema complexo. No entanto, para simplificar o tratamento da dispersão, geralmente se usa uma altura fictícia acima da fonte, esta é chamada de 'altura efetiva da chaminé': a altura da fonte é somada à altura da elevação da pluma para se calcular a altura $H_{e f}$ efetiva da emissão do poluente para a atmosfera (Equação 2).

$$
H_{e f}=h_{g}+\Delta h
$$


Onde,

$h_{g}$ é a altura física da chaminé;

$\Delta h$ é a altura de elevação da pluma em relação ao topo da chaminé.

A Figura 2 ilustra a altura efetiva da emissão do poluente, conforme a equação (2).

Figura 2 - Altura efetiva da emissão do poluente

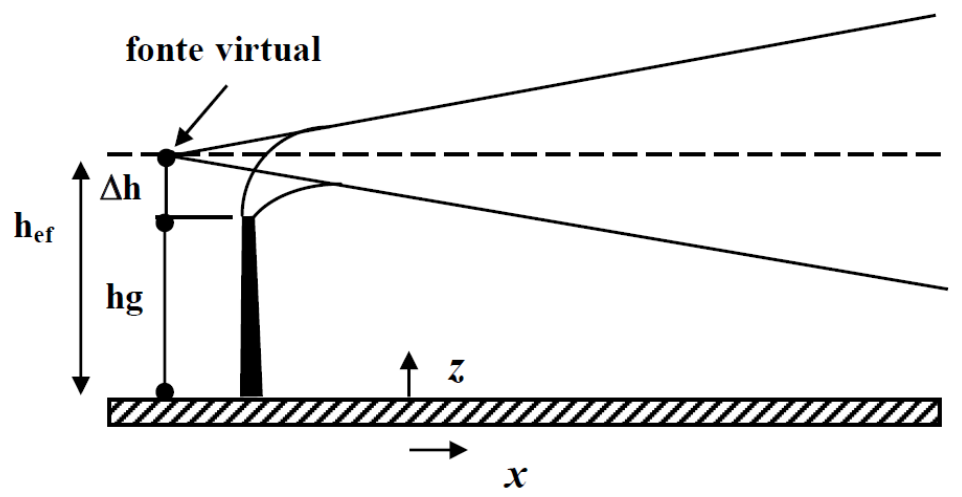

Fonte: Lisboa (2010).

Ao analisar as variáveis da Equação 1, usam-se os coeficientes de dispersão horizontal $(\sigma y)$ e vertical $(\sigma x)$. Estes podem ser estimados utilizando-se o modelo de Briggs (1974) ou de Pasquill-Gifford. O tempo de amostragem varia de 15 minutos a 1 hora e os resultados são válidos para distâncias de no máximo $10 \mathrm{~km}$. O sistema de classificação da estabilidade atmosférica mais utilizado é o de Pasquill-Gifford que define estados que vão desde o extremamente instável (Classe A) até o extremamente estável (Classe G), passando pelo neutro (Classe D). O Quadro 1 apresenta um método para se determinar a estabilidade atmosférica em função da velocidade do vento e da insolação, durante o dia, ou cobertura do céu, durante a noite (LISBOA, 2010).

Quadro 1 - Classes de Estabilidade de Pasquill-Gifford

\begin{tabular}{|c|c|c|c|c|c|c|}
\hline & $\begin{array}{l}\text { Radiaç } \\
\left(\mathrm{W} / \mathrm{m}^{2}\right.\end{array}$ & $\operatorname{lar}$ & & & $\begin{array}{l}\text { Cobertura } \\
\text { nuvens (cn }\end{array}$ & noturna de \\
\hline $\begin{array}{l}\text { Velocidade do vento } \\
10 \mathrm{~m} \text { do solo }(\mathrm{m} / \mathrm{s})\end{array}$ & $>700$ & 35 & $\leq \mathrm{I} \leq 700$ & $\mathrm{I}<350$ & $\begin{array}{l}\text { Cn } \geq \\
4 / 8\end{array}$ & cn $\leq 3 / 8$ \\
\hline$<2$ & A & $\mathrm{A}-$ & & B & - & - \\
\hline $2-3$ & $A-B$ & B & & $\mathrm{C}$ & $\mathrm{E}$ & $\mathrm{F}$ \\
\hline $3-5$ & B & $\mathrm{B}-$ & & $\mathrm{C}$ & $\mathrm{D}$ & $E$ \\
\hline $5-6$ & $\mathrm{C}$ & $\mathrm{C}-$ & & $D$ & $\mathrm{D}$ & $\mathrm{D}$ \\
\hline$>6$ & $\mathrm{C}$ & $\mathrm{D}$ & & $D$ & $\mathrm{D}$ & $\mathrm{D}$ \\
\hline $\begin{array}{l}\mathrm{A} \\
\mathrm{B} \\
\mathrm{C}\end{array}$ & $\begin{array}{l}\text { iente in } \\
\text { imente } \\
\text { te instá }\end{array}$ & & $\begin{array}{l}\text { D: neutra } \\
\text { E: franca } \\
\text { F: moder }\end{array}$ & $\begin{array}{l}\text { nente est } \\
\text { damente }\end{array}$ & & \\
\hline
\end{tabular}

Fonte: Zanetti, 1990 apud Lisboa, 2010. 
Os Quadros 2 e 3 apresentam os coeficientes de dispersão para as classes de estabilidade de Pasquill-Gifford A - F, utilizados em modelos de emissão de poluentes atmosféricos. As relações foram propostas por Briggs e são válidas para médias de 10 minutos.

Quadro 2 - Parâmetros de dispersão urbana por Briggs (para distâncias entre 100 e 10.000 m) - Média de 10 minutos

\begin{tabular}{|c|l|l|}
\hline Categoria & \multicolumn{1}{|c|}{$\sigma \mathrm{y}$} & \multicolumn{1}{c|}{$\sigma \mathrm{z}$} \\
\hline $\mathrm{A}-\mathrm{B}$ & $0,32 \times(1+0,0004 \mathrm{x})^{-0,5}$ & $0,24 \mathrm{x}(1+0,001 \mathrm{x})^{-0,5}$ \\
\hline $\mathrm{C}$ & $0,22 \mathrm{x}(1+0,0004 \mathrm{x})^{-0,5}$ & $0,20 \mathrm{x}$ \\
\hline $\mathrm{D}$ & $0,16 \mathrm{x}(1+0,0004 \mathrm{x})^{-0,5}$ & $0,14 \mathrm{x}(1+0,0003 \mathrm{x})^{-0,5}$ \\
\hline $\mathrm{E}-\mathrm{F}$ & $0,11 \mathrm{x}(1+0,0004 \mathrm{x})^{-0,5}$ & $0,08 \times(1+0,0015 \mathrm{x})^{-0,5}$ \\
\hline
\end{tabular}

Fonte: Lisboa (2010).

Quadro 3 - Parâmetros de dispersão, para condições de campo aberto, por Briggs (para distâncias entre 100 e $10.000 \mathrm{~m})$ - Média de 10 minutos

\begin{tabular}{|c|l|l|}
\hline Categoria & \multicolumn{1}{|c|}{$\sigma \mathrm{y}$} & \multicolumn{1}{c|}{$\sigma \mathrm{z}$} \\
\hline A & $0,22 \times(1+0,0001 \mathrm{x})^{-0,5}$ & $0,20 \mathrm{x}$ \\
\hline B & $0,16 \times(1+0,0001 \mathrm{x})^{-0,5}$ & $0,12 \mathrm{x}$ \\
\hline C & $0,11 \times(1+0,0001 \mathrm{x})^{-0,5}$ & $0,08 \times(1+0,0002 \mathrm{x})^{-0,5}$ \\
\hline D & $0,08 \times(1+0,0001 \mathrm{x})^{-0,5}$ & $0,06 \times(1+0,0015 \mathrm{x})^{-0,5}$ \\
\hline E & $0,06 \times(1+0,0001 \mathrm{x})^{-0,5}$ & $0,03 \times(1+0,0003 \mathrm{x})^{-1}$ \\
\hline F & $0,04 \times(1+0,0001 \mathrm{x})^{-0,5}$ & $0,016 \times(1+0,0003 \mathrm{x})^{-1}$ \\
\hline
\end{tabular}

Fonte: Lisboa (2010).

Em condições atmosféricas instáveis (classes A, B e C de Pasquill-Gifford), a concentração decresce rapidamente na pluma, uma vez que a turbulência age de modo mais eficaz do que quando a atmosfera é neutra ou estável.

Segundo Braga et al. (2005, p. 188), “o comportamento gaussiano dessa solução pode ser observado pela maneira pela qual a concentração se distribui espacialmente em relação aos eixos y e z, pelo menos enquanto as concentrações ocorrem ao longo do eixo da pluma definida por $\mathrm{y}=0 "$.

A pluma gaussiana é um modelo simplificado da realidade, passível de uma série de críticas segundo a visão teórica e a aplicação prática. Porém, o modelo tem sido aceito como uma 'ferramenta de trabalho' em estudos de avaliação e impacto ambiental, pois auxilia na ilustração comparativa de diferentes cenários de emissão poluentes. Portanto, a aplicação desse modelo deve ser considerada em função dos objetivos da análise em questão, dos recursos disponíveis e das condições locais existentes (BRAGA et al., 2005).

O estudo do processo de dispersão de poluentes na atmosfera faz uso de conceitos matemáticos, estatísticos, físicos e químicos, sendo um trabalho interdisciplinar entre as ciências básicas. Na Estatística, destaca-se o conceito da distribuição normal, também conhecida como distribuição gaussiana - conceito que o modelo tem como base. Essa distribuição tem como fundamentação o teorema central do limite - um dos alicerces do Cálculo 
Diferencial e Integral. Além disso, empregam-se, nesse modelo, os conceitos de funções de várias variáveis utilizados, também, em Cálculo Diferencial Integral, bem como em Equações Diferenciais. Na Física, pode-se explorar a teoria de Fenômenos de Transporte, tratada pela Mecânica dos Fluídos ao estudar como o meio atmosférico transporta e dispersa os poluentes nele lançados. Na Química, estudado dentro da Físico-química, analisam-se as distribuições das propriedades dos gases, os próprios modelos moleculares e a velocidade dos processos de difusão.

Ademais, pretende-se utilizar esse modelo para considerar, além das questões técnicas, matemáticas, físicas e químicas, também as questões sociocientíficas, principalmente as variáveis sociais e humanas. Para Civiero (2020), as relações de poder, subjacentes aos processos de mercantilização e industrialização, estão intimamente vinculadas ao desenvolvimento tecnocientífico, que, por sua vez, é condicionado pelos algoritmos matemáticos. Assim, defende-se que um modelo matemático precisa ser decifrado para além de seu algoritmo. É imprescindível reconhecer as variáveis que o constituem, bem como as variáveis contemporâneas que podem ser influenciadas por esse modelo.

\section{Para além das técnicas, as questões sociocientíficas: variáveis sociais e humanas}

O modelo de dispersão atmosférica discutido até o momento dá conta de revelar as potencialidades da matemática na previsão e na solução de problemas técnicos de engenharia. No entanto, intenciona-se aprofundar a discussão e chamar atenção para outros aspectos que configuram as 'condições de contorno' para a proposição de uma solução tecnológica.

O modelo em questão - e muitos outros de mesma natureza - equaciona variáveis como direção e velocidade do vento, classe de estabilidade da atmosfera, altura da fonte de poluição, taxas de emissão e superfície do solo. Desse modo, proporcionam aos profissionais de engenharia a possibilidade de simular o comportamento da pluma de dispersão de acordo com a alteração de qualquer uma de tais variáveis. As melhores decisões técnicas podem ser tomadas a partir dessas mudanças de cenário. Portanto, a engenharia cumpre com seu papel de resolver problemas, como no caso da indústria - capitalista - que no seu fazer produtivo e tecnológico causa problemas ambientais para o coletivo da sociedade.

O modelo descrito tem sua importância, e a matemática é usada como ferramenta na construção de algoritmos que facilitam a sua execução. Entretanto, esse modelo, como tantos outros, pode ser utilizado para trazer à tona discussões ambientais contundentes, concernentes às condições sociais e humanas. Portanto, é preciso desvelar as variáveis reais que compõem esse modelo e debater sobre as implicações sociais da tecnociência que estão imbricadas na resolução desse algoritmo.

Pretende-se aprofundar essa discussão com o seguinte questionamento: por que a variável 'problemas respiratórios causados por poluição atmosférica' não entra como mais um elemento na composição das condições de contorno da resolução do problema ambiental gerado pelo lançamento de poluição na atmosfera? Com dados da Secretaria de Saúde, por exemplo, fica totalmente praticável incorporar tal variável ao modelo e considerá-lo nas mais diferentes simulações que podem ser feitas para a busca da solução. Tal variável pode se revelar - como se entende que ela é - a mais importante na tomada de decisão sobre os métodos e processos de mitigação para o problema em questão.

Trabalhar nessa perspectiva é explorar ao máximo o potencial da matemática na educação em engenharia. É, inclusive, aproveitar disciplinas que, como discutido anteriormente, apresentem altos índices de retenção e evasão para problematizar a sempre 
imbricada relação entre Ciência Tecnologia e Sociedade (CTS) e para fazer efetivas aproximações entre as ciências básicas e a engenharia.

Problematizações dessa natureza corroboram a necessidade de currículos articulados em torno do enfoque CTS. Todas as disciplinas se articulam em volta do entendimento de que o fazer da engenharia tem fortes e importantes implicações no contexto social, mais que isso: as questões sociais, em geral, precisam ser os elementos definidores das soluções tecnológicas. É isso que as DCNs querem dizer quando preconizam que os profissionais de engenharia tenham capacidade de absorver e desenvolver novas tecnologias, considerando seus múltiplos aspectos - sociais, políticos, econômicos, ambientais e culturais - no atendimento às demandas e às necessidades da sociedade, quando propõe uma nova forma de conceber a educação em engenharia alicerçada em discussões consistentes e conscientes sobre as questões sociocientíficas, isto é, variáveis sociais e humanas.

As discussões feitas até esse ponto objetivam problematizar e apresentar elementos para as mudanças que se consideram urgentes na educação em engenharia. com a necessidade de entender os problemas de engenharia como equações de múltiplas variáveis, aponta-se para um processo formativo crítico, reflexivo e interdisciplinar. Quando a educação em engenharia for problematizada por meio das variáveis contemporâneas, o engenheiro poderá assumir uma 'atuação crítica', conforme mencionada nas Diretrizes Curriculares para o Ensino de Engenharia. Para tanto, faz-se premente oportunizar reflexões sociais e humanas sobre os modelos tecnocientíficos utilizados.

Em vista disso, não cabe a atitude de estudar matemática e seus algoritmos nos cursos de engenharia isoladamente. É preciso compreender as causas e os fatores reais dos quais dependem a vida cotidiana para que novos engenheiros possam contribuir para a interpretação, compreensão e transformação da realidade, preocupados com a qualidade de vida das pessoas. Por fim, é preciso entender a matemática como elemento estruturante desse processo. Refletir sobre as imbricações sociais da matemática é o primeiro passo em direção à transição para uma educação crítica e para a formação de engenheiros preocupados com as variáveis contemporâneas sociais e humanas.

\section{Referências}

BAZZO, W. A. De técnico e de humano: questões contemporâneas. 3. ed. atual., ampl. Florianópolis: Ed. da UFSC, 2019.

BAZZO, W. A.; PEREIRA, L. T. V.; LINSINGEN, I. Educação tecnológica: enfoques para o ensino de engenharia. 3. Edição. Florianópolis: Editora da UFSC, 2016.

BRAGA, B. et al. Introdução à engenharia ambiental. São Paulo: Prentice Hall, 2005.

BRASIL. Conselho Nacional de Educação. Câmara de Educação Superior. Parecer CNE/CES 1, de 23 de abril de 2019. Institui Diretrizes Curriculares Nacionais do Curso de Graduação em Engenharia. 2019. Disponível em: < http://portal.mec.gov.br/docman/marco2019-pdf/109871-pces001-19-1/file >. Acesso em: 27 jan. 2020.

CIVIERO, P. A. G. Educação Matemática Crítica e as implicações sociais da ciência e da tecnologia no processo civilizatório contemporâneo: embates para a formação de professores de matemática. Tese (Doutorado) - Universidade Federal de Santa Catarina, Florianópolis, 2016. 
CIVIERO, P. A. G. A equação civilizatória e a pertinência de uma educação insubordinada. Revista Internacional de Pesquisa em Educação Matemática - RIPEM, 2020. (no prelo)

GODOY, E. V.; ALMEIDA, E. de. A evasão nos cursos de engenharia e a sua relação com a matemática: uma análise a partir do COBENGE. Revista Educação Matemática Debate, Montes Claros, v. 1, n. 3. p. 339-361, 2017.

GOMES, E. Contribuições do método jigsaw de aprendizagem cooperativa para a mobilização dos estilos de pensamento matemático por estudantes de engenharia. Tese (Doutorado) - Pontifícia Universidade Católica, São Paulo, 2015.

GOMES, G. H. A matemática em um curso de engenharia: vivenciando culturas. Tese (Doutorado) - Pontifícia Universidade Católica, São Paulo, 2009.

GUNTHER, S. M. A matemática nos cursos superiores de engenharia mecânica em universidades paranaenses. Dissertação (Mestrado) - Universidade Tuiuti do Paraná, Curitiba, 2016.

LISBOA H. de M. Controle da Poluição Atmosférica. Florianópolis: UFSC, 2010. Disponível em: http://repositorio.asces.edu.br/handle/123456789/418. Acesso em: 25 mar. 2019.

MOREIRA, D.; TIRABASSI, T. Modelo Matemático de dispersão de poluentes na atmosfera: um instrumento técnico para a gestão ambiental. Revista Ambiente e Sociedade, São Paulo, v. 7, n. 2, p. 159-173, 2004.

REIS, P. R. dos. Uma iniciativa de desenvolvimento profissional para a discussão de controvérsias sociocientíficas em sala de aula. Revista Interacções, Lisboa, n. 4, p. 64-107, 2006.

REIS, P. R. dos. Os temas controversos na Educação Ambiental. Pesquisa em Educação Ambiental, v. 2, n. 1, p. 125-140, 2007.

SANTOS, W. L. P.; MORTIMER, E. F. Abordagem de aspectos sociocientíficos em aulas de ciências: possibilidades e limitações. Investigações em Ensino de Ciências, Porto Alegre, v. 14, n. 2, p. 191-218, 2009.

SKOVSMOSE, O. Educação Matemática Crítica: a questão da democracia. Tradução: Abgail Lins, Jussara de Loiola Araújo. Campinas, SP: Papirus, 2001.

VALLIM, M. B. R.; FARINES, E.; CURY, J.E.R. Uma estrutura curricular para um curso de engenharia de controle e automação. In: CONGRESSO BRASILEIRO DE AUTOMÁTICA, 16., 2006. Anais... Salvador, 2006.

Recebido em março de 2020.

Aprovado em maio de 2020. 